\title{
REA_16 - Measles serological diagnosis: Agreement between commercial IgM ELISA tests in a State Reference Laboratory
}

Etienne Wessler Coan ${ }^{1 *}$; Felipe Francisco Bondan Tuon ${ }^{2}$.

${ }^{1}$ LACEN PR;

${ }^{2} \mathrm{PUC} P \mathrm{PR}$.

Introduction: Among the most used methods for the serological diagnosis of measles is the search of IgM antibodies by ELISA (Enzyme Linked Immuno Sorbent Assay), which can be indirect or MAC-ELISA (IgM capture ELISA). The advantages of serology are the practicality, specificity and sensitivity and its disadvantages are interferences by heterophile antibodies and cross-reactions, and the fact that a negative result in the acute phase does not exclude the diagnosis.

Objective: To evaluate the agreement between two commercial tests (kits) for the detection of IgM antibodies against the measles virus, by indirect ELISA and MAC-ELISA methods, in relation to a characterized sample panel.

Methodology: This is an accuracy study between the indirect ELISA kit - Anti-Measles Viruses ELISA IgM (Euroimmun, Lübeck, Germany), distributed by the Ministry of Health of Brazil; and the MACELISA - Measles Virus IgM micro-capture ELISA (IBL, Hamburg, Germany), received for evaluation by the Central Laboratory of Paraná. The analysis was performed according to the manual test procedure recommended by the manufacturer. A panel of serum samples $(n=68)$ was selected based on the results of IgM serology by the indirect ELISA kit. The samples used come from the spontaneous demand of the Measles Epidemiological Surveillance Program. Complementary tests, such as RT-qPCR (Real Time Reverse Transcription Polymerase Chain Reaction) and paired serology IgG antibody by ELISA, were used to characterize the panel in four groups: A $(n=14)$ : IgM reagent, with IgG seroconversion and detectable RT-qPCR; B $(\mathrm{n}=22)$ : non-reactive IgM, with IgG seroconversion and detectable RT-qPCR; $\mathrm{C}(\mathrm{n}=11)$ : IgM reagent, without IgG seroconversion and / or RT-qPCR not detectable; D $(\mathrm{n}=21)$ : nonreactive IgM, without IgG seroconversion and non-detectable RT-qPCR. The group E $(n=20)$ complete the panel with serums reagents for: dengue virus $(n=3)$, cytomegalovirus $(n=3)$, epstein-barr $(n=$ $3)$, human parvovirus B19 $(\mathrm{n}=4)$ and COVID-19 $(\mathrm{n}=7)$. For data analysis, the kappa index $(\mathrm{k})$ was calculated and the Landis and Koch agreement scale was used to interpret the result.

Results: A median agreement $(\mathrm{k}=0.50)$ was observed between the kits. The samples from groups $\mathrm{A}$ and D were $100 \%$ concordant, while group $\mathrm{C}$ showed the lowest agreement $(4 / 11,36.6 \%)$, followed by group B $(12 / 22,54.5 \%)$. Group E showed cross reactivity to cytomegalovirus (1/3) and epstein-barr (1/3) in both kits.

Conclusion: The data indicate a moderate agreement between the evaluated kits, showing a better performance of the MAC-ELISA kit in relation to groups B and $\mathrm{C}$ of the sample panel. The relevance of this study is related to improving the diagnosis and the perspective of using earlier and more accurate serological tests.

Keywords: Measles IgM antibodies; Sero diagnosis; ELISA 